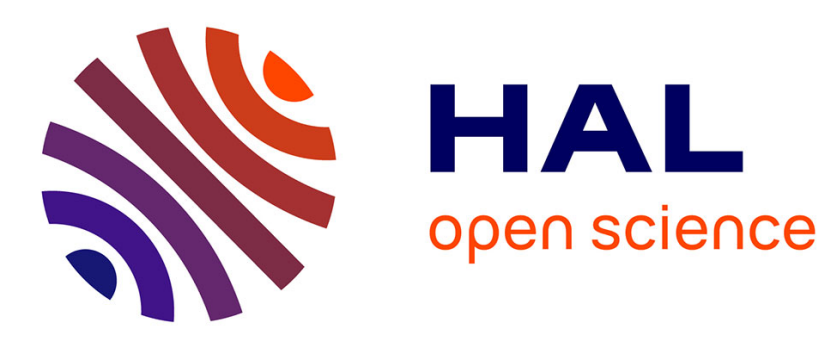

\title{
Modeling of the magnetic behavior of permanent magnets including ageing effects
}

Anderson S Nunes, Laurent Daniel, Maya Hage-Hassan, Mathieu Domenjoud

\section{To cite this version:}

Anderson S Nunes, Laurent Daniel, Maya Hage-Hassan, Mathieu Domenjoud. Modeling of the magnetic behavior of permanent magnets including ageing effects. Journal of Magnetism and Magnetic Materials, 2020, 512, pp.166930. 10.1016/j.jmmm.2020.166930 . hal-02905104

\section{HAL Id: hal-02905104 https://hal.science/hal-02905104}

Submitted on 20 Oct 2021

HAL is a multi-disciplinary open access archive for the deposit and dissemination of scientific research documents, whether they are published or not. The documents may come from teaching and research institutions in France or abroad, or from public or private research centers.
L'archive ouverte pluridisciplinaire HAL, est destinée au dépôt et à la diffusion de documents scientifiques de niveau recherche, publiés ou non, émanant des établissements d'enseignement et de recherche français ou étrangers, des laboratoires publics ou privés. 


\title{
Modeling of the magnetic behavior of permanent magnets including ageing effects
}

\author{
Anderson S. Nunes ${ }^{1,2,3}$, Laurent Daniel ${ }^{1,2}$, Maya Hage-Hassan ${ }^{1,2}$, Mathieu Domenjoud ${ }^{1,2}$ \\ ${ }^{1}$ Université Paris-Saclay, CentraleSupélec, CNRS, Laboratorie de Génie Electrique et Electronique deParis, 91192 Gif-sur-Yvette, France \\ ${ }^{2}$ Sorbonne Université, CNRS, Laboratoire de Génie Electrique et Electronique de Paris, 75252, Paris, France \\ ${ }^{3}$ ANSYS Inc., Canonsburg, PA 15317 USA
}

\begin{abstract}
In this paper, an estimation method for the ageing factor of permanent magnets is proposed. It is based on a multiscale model, which allows estimating the magnetic properties of permanent magnets, combined with the magnetic viscosity theory. Usingof this method, the necessary measurements can be drastically reduced. The results obtained are compared with measurements, with a satisfactory agreement.
\end{abstract}

Keywords: Functional fatigue, magnetic ageing, hysteresis, electrical machines, magnetization modeling, multi-scale model

\section{Introduction}

Permanent magnets play an essential role in a wide range of aerospace, medical, energy, electronics, and industrial applications [1]. In some of these applications, such as the aerospace, permanent magnets are designed to work at extreme temperatures, from -60 up to $500{ }^{\circ} \mathrm{C}$ [2, 3, 4].

Typically, the equipment is required to have a lifetime in the range of tens of years and it is well known that permanent magnets lose their magnetization with time, depending on the loading level of magnetic field [5], temperature [3, 6], and mechanical stress [7]. This phenomenon is known as magnetic ageing.

The most common way to estimate the ageing behavior of permanent magnets is by submitting the samples to high-temperatures during hundreds of hours and measuring the decay of the properties. In [8] the ageing effect in $\mathrm{NdFeB}$ magnets processed through two methods, namely HDDR (Hydrogenation - Disproportionation Desorption - Recombination) and melt-spinning was investigated. Distinct manufacturing processes were also studied in [9] which compared the magnetic behavior of thick hard magnetic films with bulk materials submitted to an infiltration process. The reversible and irreversible magnetic susceptibilities were obtained considering five magnetic operating points, allowing to obtain the ageing factor at those specific points. Furthermore, different temperatures, compacting pressures and atmospheres were considered under the open and closecircuit conditions. A closed-circuit configuration was also adopted in [10, 11, 12] to evaluate the magnetic properties behavior of magnets under a range of applied magnetic fields. Besides that, different permeance coefficients obtained by varying the magnets dimensions were considered.

However, there are some complexities inherent to real applications that make it difficult and expensive to perform tests considering all or even representative sets of variables. For instance, the working or duty cycles of electrical machines that affect the thermal and magnetic field cycles, as shown in [13]. Also, it is well known that the temperature and magnetic field, as depicted in Fig. 11, are not uniform along the volume of permanent magnets [14, 15], suggesting that the ageing factor is a local, non-uniform, quantity.

There are other factors, such as the environment where the magnets are used, since possible gases can have an impact on the demagnetization process [8] or crystallographic textures of the materials [16, 17].

Considering the difficulties mentioned above, we propose to analyze this problem by modeling the magnetic properties, reducing the need for measurements.

The first part of the problem is to model the magnetic properties of permanent magnets. One can divide the available models into two groups: descriptive and predictive. The first one encompasses the well- 


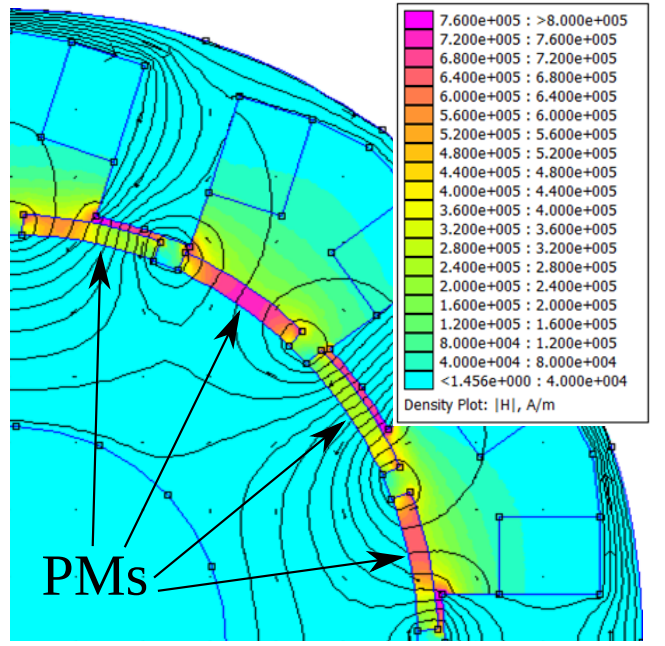

Figure 1: Magnetic field distribution inside the permanent magnets of a synchronous machine in the no-load condition.

known phenomenological mathematical models applied to describe the hysteresis [18]. However, they are not based on the physical process [19] and have no interpretation in terms of energy [20]. Such models have been extensively applied to hard magnetic materials [21, 22, 23]. In the second group, the predictive models, one can include the multi-scale approaches such as the Stoner-Wohlfarth proposed in [24] and largely applied [25, 26, 27, 28, 29, 30, 31, 32]. However, this method assumes a single or a set of uni-axial magnetically decoupled particles. Besides that, this model assumes a coherent rotation process, which is not very common in real applications, mainly in some hard magnetic materials, $\mathrm{Nd}_{2} \mathrm{Fe}_{14} \mathrm{~B}$ for instance, [33].

Thus, we propose to use a multi-scale approach based on an energetic procedure at the single crystal scale followed by a homogenization procedure to predict the macroscopic behavior of magnetic materials based on their local properties [34, 35]. Such approaches have been extensively applied to Iron-Silicon steel [36, 37, 34, 38, 35, 39, 40, 41] or giant magnetostrictive materials [42, 43], but seldom to hard magnetic materials so far.

The second part of the problem is to predict the ageing, namely, the way these local properties evolve with time, depending on the experienced thermal, magnetic or mechanical loadings. To solve this part of the problem, we use the classical magnetic viscosity theory. These ageing studies based on the activation energy concept seem to have started with [26, 44]. Then the domain wall pinning by inhomogeneities and how temperature and time, i.e. the thermal activation process, affect this phenomenon in $\mathrm{SmCo}_{5}$ alloys was presented in [45, 46, 47]. A more recent application of this theory is presented in [48].

The ageing has also been discussed for permanent magnets for some years, as shown in [3, 5, 49] for $\mathrm{SmCo}$ and in [5, 50, 51] for NdFeB magnets. Furthermore, some recent experimental works [8, 10, 11, 12, 9, 52] have been developed. Also the magnetic viscosity effect on the coercive field has been investigated in [53, 54] in order to increase the performance of the permanent magnets.

By combining the multi-scale approach with the ageing theory, it is possible to predict the magnetic behavior of permanent magnets as well as estimating the ageing factor. The proposed method can be embedded in numerical methods such as the Finite Element Method in order to estimate the ageing factor of permanent magnets inserted in real applications. A similar approach has been presented in [55], but it is based on the Stoner-Wohlfarth model, which is well adapted to independent magnetic particles and offers less flexibility for describing the magnetic behavior of inhomogeneous structures of real magnets [33].

To validate the proposed approach, the modeling results for ferrite and $\mathrm{NdFeB}$ are compared with measurements. Then, the ageing factor of a real permanent magnet at a given operating point is estimated.

\section{Multi-scale approach}

The multi-scale approach proposed in [34, 35] considers three different scales, namely :

- The magnetic domain scale, denoted by an index $\alpha$, is the scale where all magnetic properties are uniform.

- The single-crystal scale, denoted by an index $g$ is the scale where elastic properties are uniform.

- The Representative Volume Element (RVE) scale, denoted by an index $m$, is the macroscopic scale at which the material response is measured.

The multi-scale model is based on expressing the energy balance at the single-crystal level considering each single-crystal as a set of magnetic domains. Then, the RVE is approximated as an aggregate of single-crystals (polycrystal) oriented following an orientation distribution function corresponding to the crystallographic texture of the material.

This approach was developed to predict the magnetoelastic behavior of magnetic materials submitted to 
multi-axial mechanical loadings [56], but in this work, no stress is applied and mechanical effects are neglected.

\subsection{Single-crystal model}

At the single-crystal scale, the material is divided into a set of domain families. Each domain family gather all domains with the same magnetization orientation $\alpha$. The magnetization $\mathbf{M}_{\alpha}$ of each family can be expressed as:

$$
\mathbf{M}_{\alpha}=M_{s} \alpha=M_{s}\left[\alpha_{1} \alpha_{2} \alpha_{3}\right]
$$

where $M_{s}$ is the saturation magnetization and $\alpha_{1,2,3}$ are the cosines of the magnetization vector, Fig. 2 .

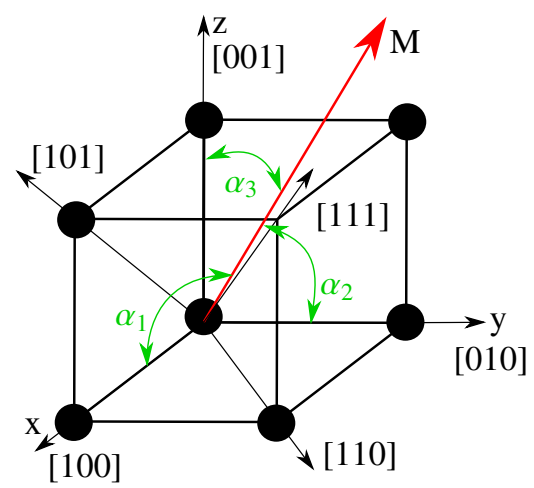

Figure 2: Representation of the magnetization vector in the single crystal frame considering a cubic anisotropy.

The magnetic equilibrium is given by the competition of the following energy terms forming the local free energy:

$$
W_{\alpha}=W_{\alpha}^{m a g}+W_{\alpha}^{a n}+W_{\alpha}^{m e c}
$$

The magnetostatic energy (or Zeeman energy), $W_{\alpha}^{\text {mag }}$, tends to align the local magnetization $\mathbf{M}_{\alpha}$ with the applied field H. $\mu_{0}$ is the vacuum magnetic permeability.

$$
W_{\alpha}^{m a g}=-\mu_{0} \mathbf{H} \cdot \mathbf{M}_{\alpha}
$$

The anisotropy energy $W_{\alpha}^{a n}$ describes the existence of preferred orientations for the magnetization (easy axes).

$$
W_{\alpha}^{a n}=K_{1}\left(\alpha_{1}^{2} \alpha_{2}^{2}+\alpha_{2}^{2} \alpha_{3}^{2}+\alpha_{3}^{2} \alpha_{1}^{2}\right)+K_{2}\left(\alpha_{1}^{2} \alpha_{2}^{2} \alpha_{3}^{2}\right)
$$

where $K_{1,2}$ are the magnetocrystalline anisotropy constants.
The magneto-elastic energy $W_{\alpha}^{\text {mec }}$ describes the effect of an applied mechanical stress on the magnetic equilibrium [34]. Since no stress will be considered in this study, this term is uniformly taken as zero.

Knowing the free energy $W_{\alpha}$ for all domain families, the volume fraction $f_{\alpha}$ of each domain family - corresponding to the existence probability of the orientation $\alpha$ for the local magnetization - can be defined using a Boltzmann-type equation [57, 58]:

$$
f_{\alpha}=\frac{\exp \left(-A_{s} W_{\alpha}\right)}{\int_{\alpha} \exp \left(-A_{s} W_{\alpha}\right) \mathrm{d} \alpha}
$$

where $A_{s}$ is defined from the initial anhysteretic susceptibility $\chi_{0}$ of the material [34]:

$$
A_{s}=\frac{3 \chi_{0}}{\mu_{0} M_{s}^{2}}
$$

$\chi_{0}$ can be obtained from the anhysteretic magnetization curve or estimated at the coercive field $H_{c}$ of a major loop $\left(\chi_{0}=\Delta M /\left.\Delta H\right|_{H=H_{c}}\right)$ [59].

Once the volume fractions are obtained for all domain families in a crystal, the average magnetization $\mathbf{M}_{g}$ at the single crystal scale can be obtained:

$$
\mathbf{M}_{g}=\int_{\alpha} f_{\alpha} \mathbf{M}_{\alpha} \mathrm{d} \alpha
$$

From a practical perspective, the solution of the single-crystal model can be achieved in three steps:

Step 1 - Calculate the total energy (Eq. 2) for a set of families $\alpha$ covering the orientations in space

Step 2 - Obtain the volume fraction for each domain family (Eq. 5)

Step 3 - Calculate the magnetization $\mathbf{M}_{g}(\mathrm{Eq} 7 \mathrm{7})$ of the single-crystal

In order to cover the possible orientations for the domain magnetization, it necessary to build a set of orientations distributed as uniformly as possible along the shell of a unit sphere. This distribution should be as homogeneous as possible to avoid possible artefact anisotropy due to inhomogeneity in the directions disposition. For this purpose the nodes of an icosphere can be used [43, 60]. An example containing 2562 directions is shown in Fig. 3 .

Regarding the computation effort, indeed the lower the number of points, the lower the computational time and effort to solve the problem. Additionally, simplified methods with a lower number of orientations, as presented in [56, 60] for instance, have been successfully tested. Another noticeable numerical aspect is the 


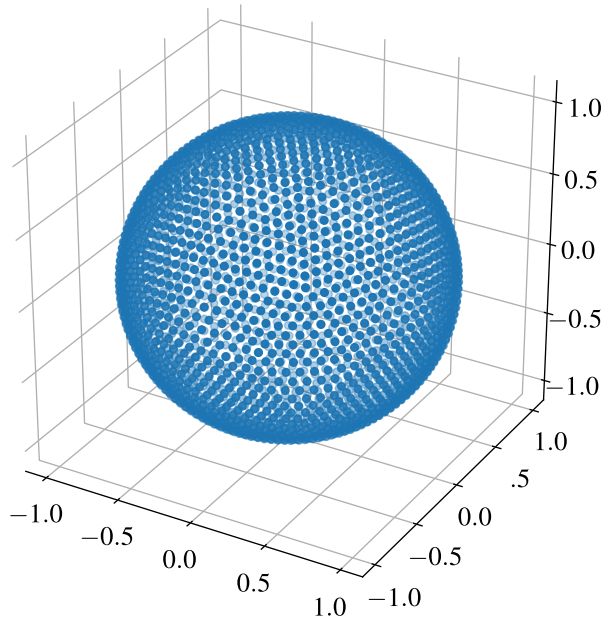

Figure 3: Unit sphere showing the distribution of the families $\alpha$ (2562 points).

straightforward parallelization of the single-crystal calculations. A method capable of quantifying the quality of a given distribution was proposed in [43], where the authors evaluated and applied a mesh composed by 10242 nodes (each node of the mesh refers to one $\alpha$ family direction). Once that distribution (10242 nodes) has been validated, it is used in this paper to obtain all the presented results.

Considering that the energy has to be calculated for all those directions, one can easily visualize the energy distribution. Fig. 4 shows the total energy landscapes for three different values of the applied magnetic field along the $<110>$ direction as a function of the anisotropy field $H_{a}$ given in Eq. 13. The considered material is a ferrite, see material parameters in Table 1 One can identify the six easy axes (low energy regions) and it is possible to verify how the energy changes when a magnetic field is applied. In particular it can be seen that the field, if high enough, rotates the magnetization out of the easy axes.

Table 1: Properties of ferrite [33].

\begin{tabular}{cccc}
\hline \hline $\begin{array}{c}\text { Parameter } \\
\text { Unit }\end{array}$ & $\begin{array}{c}M_{s} \\
{[\mathrm{~A} / \mathrm{m}]}\end{array}$ & $\begin{array}{c}K_{1} \\
{\left[\mathrm{~J} / \mathrm{m}^{3}\right]}\end{array}$ & $\begin{array}{c}K_{2} \\
{\left[\mathrm{~J} / \mathrm{m}^{3}\right]}\end{array}$ \\
\hline Value & $0.382 \times 10^{6}$ & $330 \times 10^{3}$ & 0 \\
\hline \hline
\end{tabular}

\subsection{Polycrystal}

In order to obtain the macroscopic properties of a RVE of a material, it is necessary to describe a volume large enough, i.e. containing enough single crystal orientations, to represent the average properties.

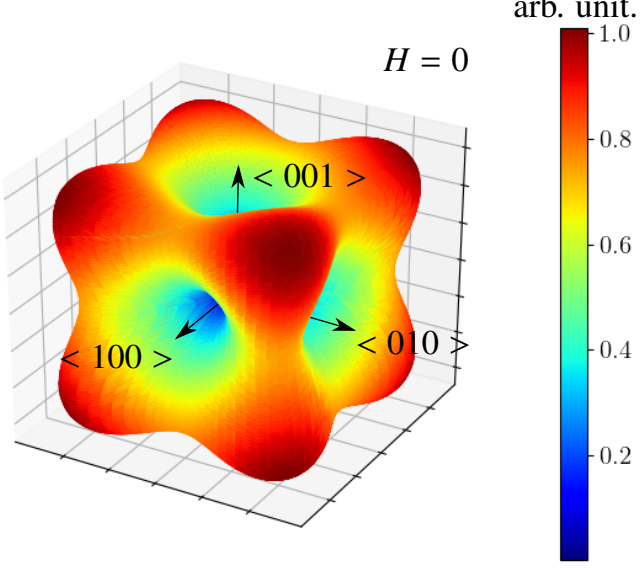

arb. unit.

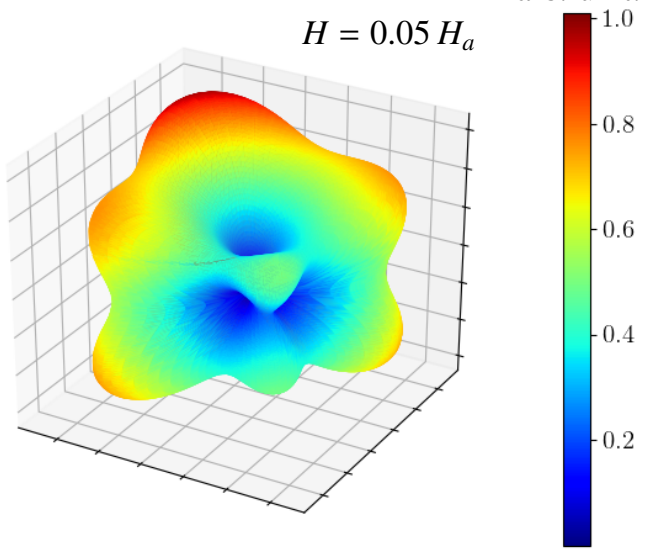

arb. unit.

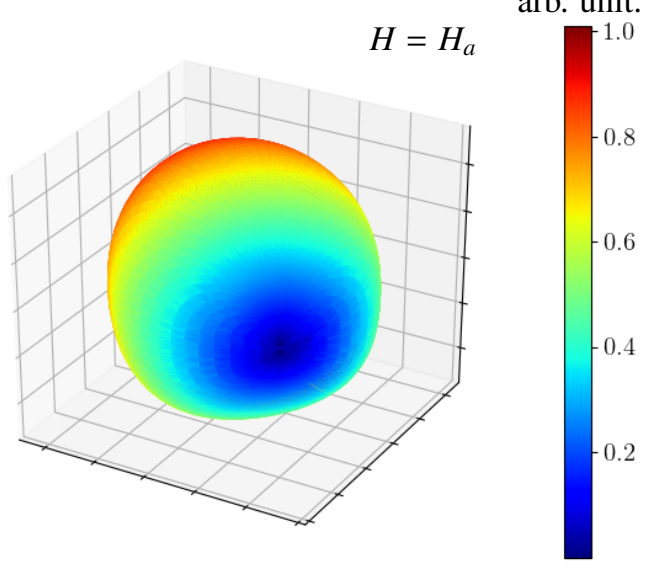

Figure 4: Total energy distribution for three different values of the applied magnetic field. $H_{a}$ is presented in Eq. 13

The crystallographic texture can be responsible for macroscopic anisotropy effects [16, 61]. It can be represented in a discrete fashion. In this study, an isotropic 
texture is used. It is built thanks to a regular zoning of the crystallographic orientations space, as already applied in [34, 58], in terms of the Euler angles [62] presented in Table 2.

Table 2: Euler angles distribution for an isotropic crystallographic texture.

\begin{tabular}{ccc}
\hline \hline Variable & Domain & Number of values \\
\hline$\Phi_{1}$ & {$[0,2 \pi]$} & 13 \\
$\cos \Phi$ & {$[0,1]$} & 7 \\
$\Phi_{2}$ & {$[0,2 \pi]$} & 6 \\
\hline \hline
\end{tabular}

In order to calculate the macroscopic properties of the sample, it is required to express the macroscopic loading (applied magnetic field $\mathbf{H}$ ) at the local scale [34, 43]. In this paper, we assume uniform magnetic field within the material so that the single crystal is subjected directly to the applied magnetic field $\mathbf{H}$. From this local loading, the single crystal magnetization $\mathbf{M}_{g}$ of each single crystal ( $\mathrm{N}=13 \times 7 \times 6=546$ orientations) is calculated according to the single crystal model.

Finally, the average magnetization of the material is obtained by averaging the local magnetization over all orientations:

$$
\mathbf{M}_{m}=\frac{1}{N} \sum_{i=1}^{N} \mathbf{M}_{g}
$$

\subsection{Irreversible contribution}

The modelling approach is anhysteretic so far, i.e., it takes into account only the reversible part of the magnetic behavior. Describing the complete hysteresis cycle of permanent magnets implies both reversible and irreversible parts to be modelled. In this case the Hauser's method is used [35, 63, 64] in order to estimate the irreversible contribution on the magnetic field $\mathbf{H}_{i r r}$,

$$
\left|\mathbf{H}_{i r r}\right|=\delta\left(\frac{k_{r}}{\mu_{0} M_{s}}+c_{r}|\mathbf{H}|\right)\left[1-k_{g} \exp \left(-\frac{k_{a}}{k_{g}}\right)\left|\mathbf{M}-\mathbf{M}^{r e b}\right|\right]
$$

where $\delta$ is equal to \pm 1 depending on whether the applied magnetic field is increasing or decreasing, $c_{r}$ and $k_{g}$ are material parameters, and

$$
k_{r}=\mu_{0} M_{s} H_{c} .
$$

Based on [64], $k_{a}$ is expressed by

$$
k_{a}=\beta \frac{H_{a}}{H_{c}},
$$

where

$$
\beta=\frac{\mu_{0} M_{s}^{2}}{2 \chi_{0} K_{1}}
$$

and the anisotropy field $H_{a}$ is given by

$$
H_{a}=\frac{2 K_{1}}{\mu_{0} M_{s}}
$$

Following the magnetization process in time, $k_{g}$ is updated at each return point depending on its previous value, $k_{g}^{\prime}$,

$$
k_{g}=2-k_{g}^{\prime} \exp \left(-\frac{k_{a}}{k_{g}^{\prime}}\right)\left|\mathbf{M}-\mathbf{M}^{r e b}\right|
$$

$\mathbf{M}^{r e b}$ is the magnetization at the previous inversion of the magnetic field, which can be detected by checking $\Delta \mathbf{H}(t) \times \Delta \mathbf{H}(t-\Delta t)<0$.

This model depends on the coercive field $H_{c}$. However, $H_{c}$ is a temperature-dependent quantity [2, 54, 53, 65]. At high temperatures the effects of thermal activation on the reversal process are increased, reducing the coercivity [54]. It is explained by the influence of temperature on the energy-barriers, which depend on $K_{B} T$, where $K_{B}$ is the Boltzmann constant and $T$ is the temperature. Consequently, the proposed method becomes temperature-dependent as well.

\section{Ageing phenomena}

If the magnetic field acting on a magnetic material changes, the magnetization will keep evolving over a certain time longer than the short times associated with the eddy current effects [47]. This time dependence of the magnetic properties, such as magnetization, is known as magnetic viscosity or ageing [66].

This behavior is caused by thermal activation of magnetization reversals over the energy barriers and the waiting time before an energy barrier is overcome is referred to as the relaxation time $\tau$, which is given by [47]:

$$
\tau^{-1}=C \exp \left(\frac{E_{a}}{K_{B} T}\right)
$$

where $E_{a}$ is the activation energy of a given energy barrier and $C$ is a frequency constant of order $10^{10}-10^{12}$ $\mathrm{Hz}$.

As can be noticed from Eq. 15, the energy landscape is key to the ageing behavior. This energy landscape highly depends on the microstructure of the material [67]. The energy landscape models are often based on the assumption of single-domain particles, as presented in [48], which may not describe the pinning effects in permanent magnet materials. 
It was observed experimentally [26] that the magnetization changes linearly with the natural logarithm of time. This decay can be described by [49,68]

$$
M(t)=M\left(t_{0}\right)-S \ln \left(\frac{t}{t_{0}}\right)
$$

where

$$
S=2 M_{s} K_{B} T f\left(E_{a}\right)
$$

$f\left(E_{a}\right)$ is an energy-barrier distribution which depends on the activation energy $E_{a}$.

In order to separate the field-dependent irreversible susceptibility $\chi_{\text {Irr }}$ from the magnetic viscosity problem, it is convenient to express $S$ as [33]:

$$
S=\frac{K_{B} T \chi_{\text {Irr }}}{v_{a} \mu_{0} M_{s}}
$$

where $v_{a}$ is the activation volume.

Thus, the magnetic viscosity coefficient $S_{v}$ is defined as

$$
S_{v}=\frac{K_{B} T}{v_{a} \mu_{0} M_{s}}
$$

$S_{v}$ is shape independent, unlike $\chi_{\text {Irr }}$ and $S$ [50]. $S$ can finally be expressed as:

$$
S=S_{v} \chi_{\text {Irr }}
$$

The magnetic viscosity is attributed to irreversible changes of the magnetization across the energy barriers [53, 69], thus it affects only the irreversible variation of the magnetization that can be described by the irreversible susceptibility $\chi_{\text {Irr }}\left(\chi_{\text {Irr }}=\chi_{\text {Tot }}-\chi_{\text {Rev }}\right)$. This parameter can be obtained experimentally following the procedures presented in [5, 9].

Considering the above-presented formulae and that the activation energy, Eq. 17, depends on the material properties discussed in Section 2, one can find out that the magnetic ageing depends on the material properties, applied magnetic field vector, time, and temperature.

This ageing methodology is applied based on the following steps:

Step 1 - Calculate the irreversible susceptibility $\left(\chi_{\text {Irr }}=\right.$ $\left.\chi_{T o t}-\chi_{\text {Rev }}\right)$ for a given number of operating points. This can be done from small loops included in a major loop. The major loop provides $\chi_{T o t}$ at a given excitation level while the slope of the minor loop provides $\chi_{\text {Rev }}$

Step 2 - Obtain the $S$ parameter for the same operating points. This is done by measuring the change in magnetization over time at a given fixed excitation. $S$ is then obtained using Eq. 16
Step 3 - Express $S$ as a function of the applied magnetic field. It can be done in two different ways:

(a) Interpolate $S$ and apply it directly into Eq. 16 for any time and magnetization.

(b) Calculate $S_{v}$ (Eq. 20) and then $v_{a}$ (Eq. 19. Interpolate $v_{a}$. The $S$ parameter is then obtained by Eq. 18

The procedures for steps one and two are detailed in [9]. It was assumed that the applied magnetic field and temperature are constant over time.

\section{Experimental setup}

A specific set up was designed and built to investigate the magnetic properties of permanent magnets.

The standard [70] suggests a magnetic circuit with one air gap, but in this work, the magnetic circuit is composed of two U-shaped Fe-Si yokes to create two air gaps, and four excitation coils, as depicted in Figures 5 and 6. Therefore two magnets are characterized at the same time, one at each air gap of the device, which helps to ensure the robustness of the measurements. The depth of the magnetic yokes is $52 \mathrm{~mm}$.

The dimensions of the transverse section of the magnetic circuit are chosen to impose a magnetic field as uniform as possible in the air gaps.

The magnetic induction is measured by a coil wounded around each magnet and the magnetic field in the air gaps is measured by two Brockhaus BGM 201 gaussmeters with two transverse probes BTP 201-F75.

The control of the applied current and the acquisition of the signals are performed by a dSPACE processor board DS 1006 connected to a computer. The A/D and D/A boards are, respectively, the DS2004 and the DS2103.

More information about the control system is available in [71], also providing information about demagnetization process, drift in B, current control and acquisition system. 


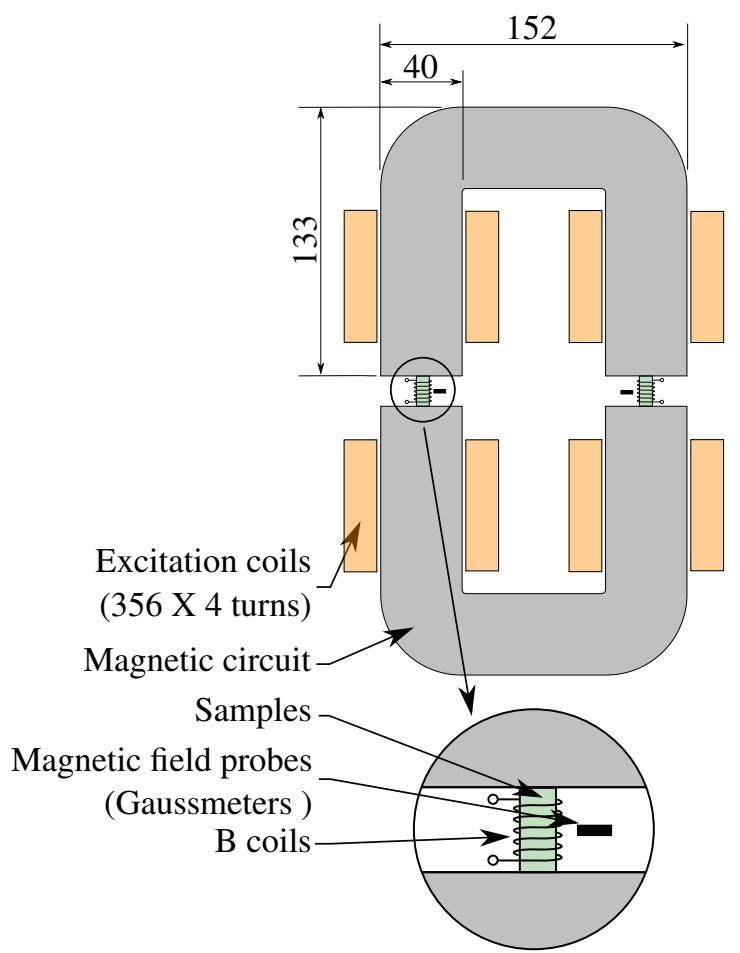

The dimensions are in $\mathrm{mm}$.

Figure 5: Experimental setup schematic.

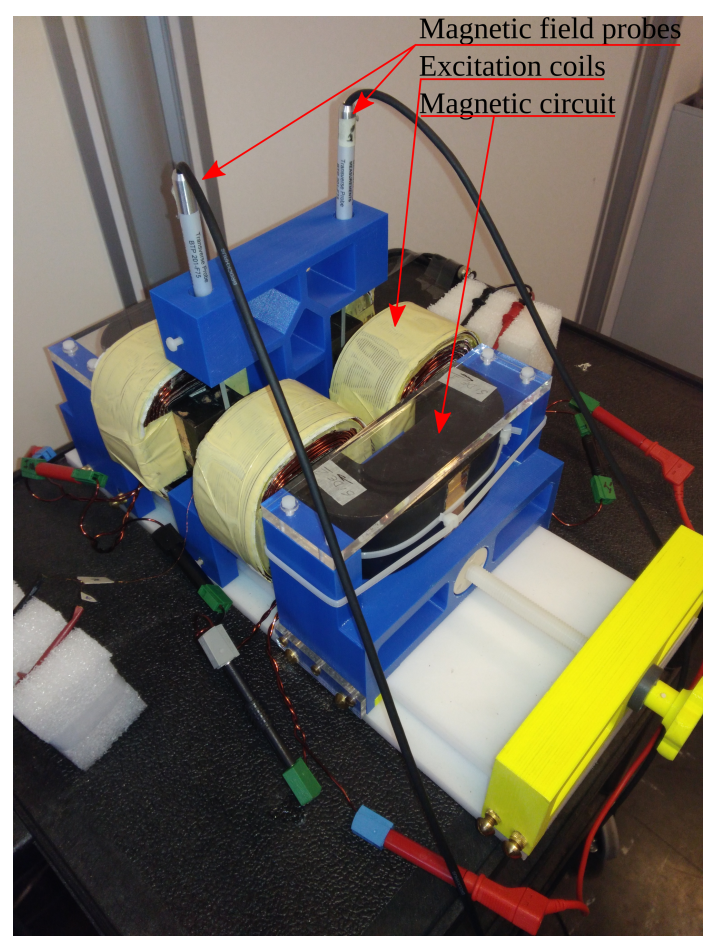

Figure 6: Picture of the experimental setup.
The experimental setup is used to measure the hysteresis loops of the permanent magnets and, based on these loops, the saturation magnetization $M_{s}$, the coercive field $H_{c}$, and the initial susceptibility $\chi_{0}$.

$M_{s}$ is the maximum measured magnetization, $H_{c}$ is obtained at the intersection of the curve with the $x$ axis, i.e., $M=0$, and $\chi_{0}$ is estimated by $\left.\frac{\Delta M}{\Delta H}\right|_{H=H_{c}}$ [59].

The applied signal is sinusoidal at $2 \mathrm{~Hz}$ frequency (controlled in current).

\section{Results}

First, the results obtained with the multi-scale model are presented for two different materials, namely ferrite and $\mathrm{NdFeB}$.

Then, the measurement data given in [9] are used to obtain the ageing parameters mentioned in Section 3 and the results obtained with this procedure are presented.

\subsection{Multi-scale approach}

\subsubsection{Ferrite}

In this case, the material parameters were obtained using the in-house measurement setup presented in Section 4, except for $K_{1}$ which was found in [33], and are presented in Table 3

Table 3: Properties of ferrite.

\begin{tabular}{ccccc}
\hline \hline Parameter & $M_{s}$ & $K_{1}$ & $\mathrm{H}_{c}$ & $\chi_{0}$ \\
Unit & {$[\mathrm{A} / \mathrm{m}]$} & {$\left[\mathrm{J} / \mathrm{m}^{3}\right]$} & {$[\mathrm{A} / \mathrm{m}]$} & - \\
\hline Value & $0.416 \times 10^{6}$ & $330 \times 10^{3}$ & $257 \times 10^{3}$ & 50 \\
\hline \hline
\end{tabular}

The anhysteretic curve modeled with the multi-scale approach (Sections 2.1 and 2.2), the hysteresis cycle obtained by adding the reversible field portion (Section 2.3 and the measurement data are shown in Fig. 7

A good match between the results is obtained, except along the "knees" in the upper-right and lower-left curves. Nonetheless, typically in the case of permanent magnets, the second-quadrant "knee" is used and along this region, the model presents a good correlation with measurements. 


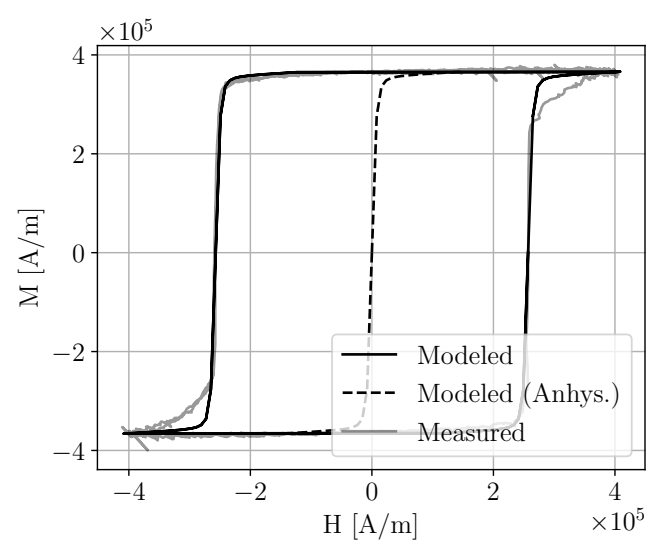

Figure 7: Comparison between the multi-scale model and the measurement for the ferrite.

\subsection{2. $\mathrm{NdFeB}$}

The same procedure is followed for the $\mathrm{NdFeB}$ material, whose properties are given in [9] and presented in Table 4. The results are shown in Fig. 8. given in the three steps, with sub-item (b) presented in Section 3 .

The $\mathrm{S}$ factor and the $\chi_{0}$ measured are presented in Figures 9 and 10 . It is possible to see the similarity between these figures. It is due to the irreversible characteristic of the ageing factor $S$ [44].

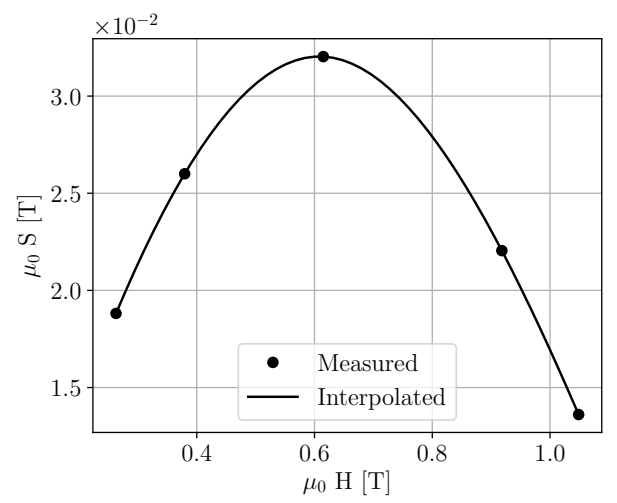

Figure 9: S factor interpolated from [9].

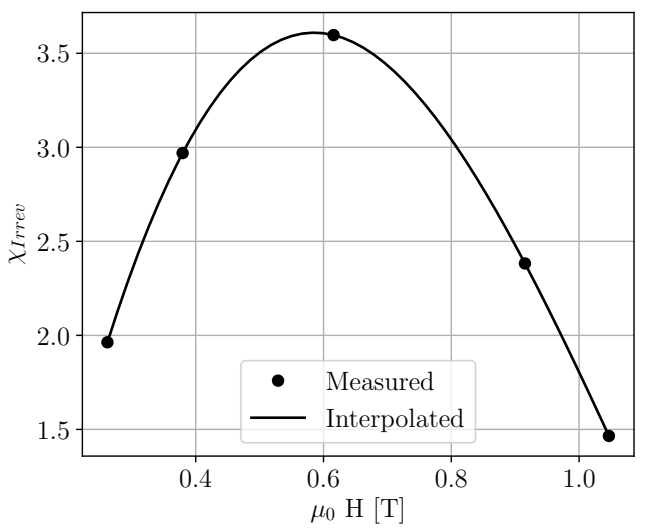

Figure 10: Irreversible susceptibility interpolated from [9].

From Eq. 18 it is possible to obtain the activation volume $v_{a}$, which is an important parameter since smaller the volume, more susceptible to thermal degradation the material is [55]. For this specific case and temperature $(400 \mathrm{~K})$, from Fig. 11 one can see that $v_{a}$ does not vary drastically in terms of the applied field, suggesting that it would be reasonable to assume it as a constant.

With the second quadrant of the hysteresis loop depicted in Fig. 8 and by interpolating these ageing parameters, it is possible to estimate the decay of magnetization in time for any value of the magnetic field for the complete working range of the permanent magnet. 
An example is depicted in Fig. 12, where the second quadrant of the hysteresis loops are compared and one random operating point is chosen. In this case, it is possible to estimate that for this given $\mathrm{Nd}_{2} \mathrm{Fe}_{14} \mathrm{~B}$ at $400 \mathrm{~K}$ and after 2000 hours, the magnetization will be reduced to about $85 \%$ of its initial value.

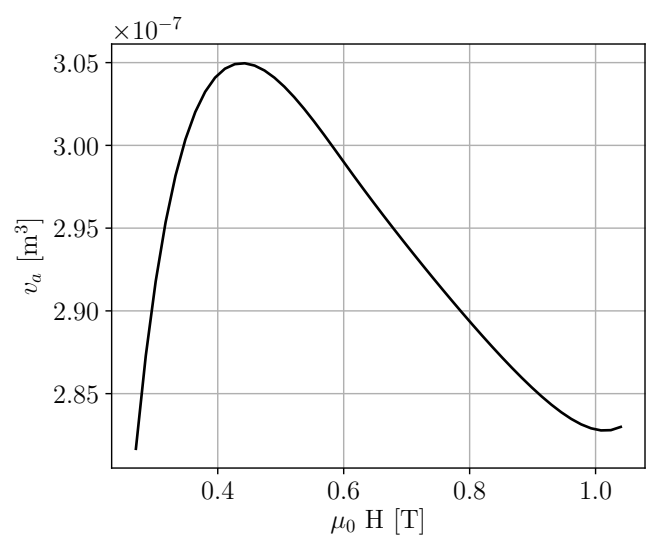

Figure 11: Activation volume.

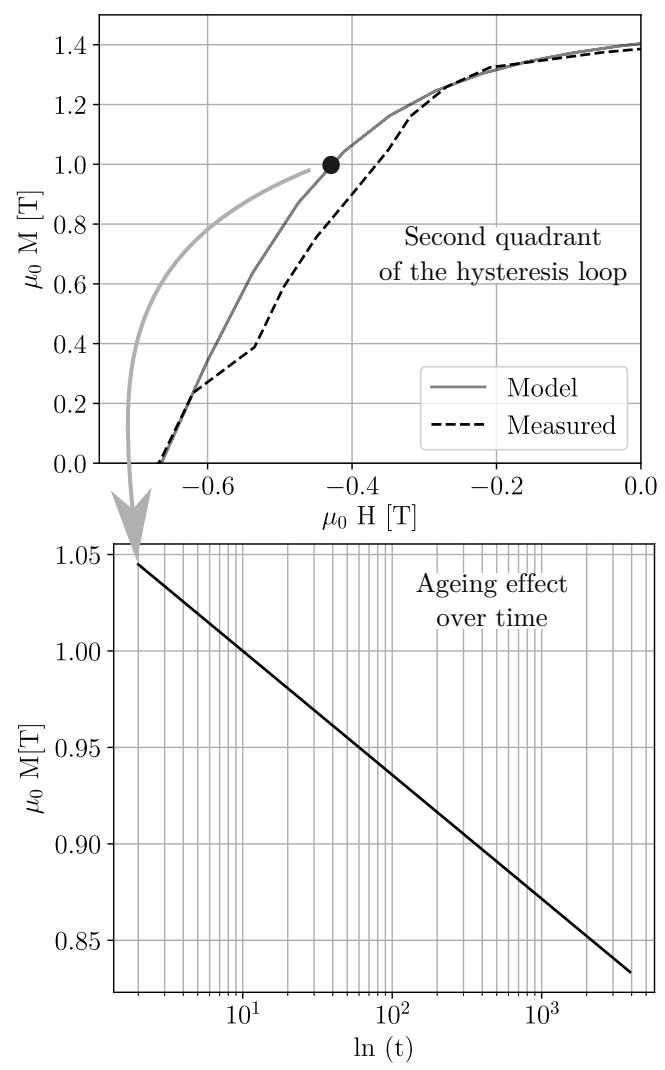

Figure 12: Decay of magnetization in time (ageing).

\section{CONCLUSION}

A multi-scale approach was used to describe the macroscopic behavior of permanent magnets. It was shown that this approach, using only four to five material parameters, was able to describe with satisfactory accuracy the major hysteresis loops of ferrite and $\mathrm{Nd}$ FeB magnets.

Then, an elementary but well-established ageing model was used. The model is based on the measurement of two parameters, namely the ageing factor and the irreversible susceptibility, for a set of magnetic excitations.

Combining the two approaches proved to be an efficient way to estimate the ageing of permanent magnets working at any magnetic operating point. It was shown for instance that a $\mathrm{NdFeB}$ magnet placed at $400 \mathrm{~K}$ during $2000 \mathrm{~h}$ experiences a reduction of magnetization of about $15 \%$.

It was also found that the ageing factor appears to be proportional to the irreversible susceptibility, which means that the $S$ factor reaches its maximum at the coercive field.

The proposed approach is a first attempt to describe in a predictive way ageing effects in permanent magnets. It is restricted so far to constant magnetic field and temperature loadings. The effect of stress was not considered in this study, but it is naturally incorporated in the multi-scale approach. Experimental validation, however, would be required. The study also considered isotropic magnets, but the multi-scale approach provides a straightforward way to take into account crystallographic textures through the description of grain orientations. The effect of the operating environment and notably aggressive gases can also be significant and was not studied here.

The proposed material model is a first brick to the development of ageing models. Indeed, practical devices are subjected to external loadings significantly varying in time. Scaling rules are required to predict the evolution of magnetization under such variable loadings from the knowledge of the response under constant loadings. This latter point is still a very open field of research.

\section{Acknowledgment}

This work was supported by the Automotive Mechatronics Chair, a cooperation between Faurecia, CentraleSupélec and Esigelec. 


\section{References}

[1] GVR, Permanent magnet market size, share \& trend report permanent magnet market size, share \& trend analysis report by product (ferrite magnet, rare earth), by application (automotive, electronics, industrial) by region, and segment forecasts, 2018 2024 Eletronic (Mar. 2018).

URL https://www.grandviewresearch.com/ industry-analysis/permanent-magnets-industry

[2] H. Feng, H. Chen, Z. Guo, W. Pan, M. Zhu, W. Li, Investigation on microstructure and magnetic properties of sm2co17 magnets aged at high temperature, Journal of Applied Physics 109 (7) (2011) 07A763. doi:10.1063/1.3563084

[3] S. Liu, E. Hoffman, J. Brown, Long-term aging of $\mathrm{Sm} 2(\mathrm{Co}, \mathrm{Fe}, \mathrm{Cu}, \mathrm{Zr}) 17$ permanent magnets at 300 and $400 \mathrm{C}$, IEEE Transactions on Magnetics 33 (5) (1997) 3859-3861. doi:10.1109/20.619595

[4] J. R. Hendershot Jr., T. J. E. Miller, Design of Brushless Permanent-Magnet Motors, Magna Physics Publishing and Oxford University Press, 1994.

[5] R. Street, R. Day, J. Dunlop, Magnetic viscosity in NdFeB and SmCo5 alloys, Journal of Magnetism and Magnetic Materials 69 (1) (1987) 106-112. doi:10.1016/0304-8853(87) 90217-4

[6] A. Lekdim, L. Morel, M. Raulet, Effect of the remaining magnetization on the thermal ageing of high permeability nanocrystalline FeCuNbSiB alloys, Journal of Magnetism and Magnetic Materials 460 (2018) 253-262. doi:10.1016/j.jmmm.2018. 03.051

[7] M. Mito, H. Goto, K. Nagai, K. Tsuruta, H. Deguchi, T. Tajiri, K. Konishi, High pressure effects on isotropic nd2fe14b magnet accompanying change in coercive field, Journal of Applied Physics 118 (14) (2015) 145901. doi:10.1063/1.4932599

[8] L. U. Lopes, Caracterização da estabilidade térmica das propriedades magnéticas de ímãs compósitos à base de Nd2Fe14B, PhD Thesis, Universidade Federal de Santa Catarina, Centro Tecnológico (2008)

[9] G. Ciuta, Une étude expérimentale de la coercivité des aimants $\mathrm{NdFeB}, \mathrm{PhD}$ Thesis, Université de Grenoble (2013).

[10] M. Haavisto, Studies on the time-dependent demagnetization of sintered NdFeB permanent magnets, PhD Thesis, Tampere University of Technology, Tampere (2013).

[11] M. Haavisto, M. Paju, Time-dependent demagnetization in sintered NdFeB magnets, in: Proceedings of 21st Workshop on Rare-Earth Permanent Magnets and Their Applications, Bled, Slovenia, 2010.

[12] M. Haavisto, S. Tuominen, T. Santa-Nokki, H. Kankaanpää, M. Paju, P. Ruuskanen, Magnetic behavior of sintered NdFeB magnets on a long-term timescale, Advances in Materials Science and Engineering 2014 (2014) 1-7. doi:10.1155/2014/ 760584

[13] D. Huger, D. Gerling, The effects of thermal cycling on aging of neodymium-iron-boron magnets, in: 2015 IEEE 11th International Conference on Power Electronics and Drive Systems, IEEE, 2015. doi:10.1109/peds . 2015.7203464

[14] S. Ruoho, A. Arkkio, Partial demagnetization of permanent magnets in electrical machines caused by an inclined field, IEEE Transactions on Magnetics 44 (7) (2008) 1773-1778. doi: 10.1109/tmag. 2008.921951

[15] C. Gutfrind, Electromechanical actuators optimization used in thermal engine air flow regulation PhD Thesis, Supélec (Jul. 2012). tel-00966937

[16] C. G. Hauegen, F. R. F. da Silva, F. A. S. da Silva, J. F. C. Lins,
M. F. de Campos, EBSD texture analysis of $\mathrm{NdFeB}$ magnets, Materials Science Forum 727-728 (2012) 135-139. doi:10. 4028/www.scientific.net/msf .727-728.135

[17] K. Song, Y. Fang, S. Wang, N. Yu, H. Chen, M. Zhang, M. Zhu, W. Li, Crystalline and magnetic microstructures of iron-rich $\mathrm{Sm}\left(\mathrm{Co}_{0.65} \mathrm{Fe}_{0.26} \mathrm{Cu}_{0.07} \mathrm{Zr}_{0.02}\right)_{7.8}$ sintered magnets: Isothermal aging effect, Journal of Magnetism and Magnetic Materials 465 (2018) 569-577. doi:10.1016/j.jmmm.2018.06.047

[18] I. Mayergoyz, Mathematical Models of Hysteresis and Their Applications, Elsevier, 2003. doi: 10.1016/B978-0-12-480873-7.50009-8

[19] C. Serpico, C. Visone, Magnetic hysteresis modeling via feedforward neural networks, IEEE Transactions on Magnetics 34 (3) (1998) 623-628. doi:10.1109/20.668055

[20] V. François-Lavet, F. Henrotte, L. Stainier, L. Noels, C. Geuzaine, An energy-based variational model of ferromagnetic hysteresis for finite element computations, Journal of Computational and Applied Mathematics 246 (2013) 243-250. doi:10.1016/j.cam.2012.06.007

[21] M. Rosu, T. Jokinen, Simulation of the magnetic hysteresis in nfeb permanent magnet using preisach's model, in: Proceedings of the 6th International Conference on Optimization of Electrical and Electronic Equipments, 1998. OPTIM '98., Vol. 36, Institute of Electrical and Electronics Engineers (IEEE), 1998, pp. 418-428. doi:10.1109/OPTIM.1998.710431

[22] E. Torre, L. Bennett, R. Fry, O. Ducal, Preisach-arrhenius model for thermal aftereffect, IEEE Transactions on Magnetics 38 (5) (2002) 3409-3416. doi:10.1109/tmag. 2002.802702

[23] D. R. Cornejo, F. P. Missell, Preisach analysis of reversible magnetization in SmCo/fe films, Journal of Applied Physics 87 (9) (2000) 4741-4743. doi:10.1063/1.373144

[24] E. C. Stoner, E. P. Wohlfarth, A mechanism of magnetic hysteresis in heterogeneous alloys, Philosophical Transactions of the Royal Society A: Mathematical, Physical and Engineering Sciences 240 (826) (1948) 599-642. doi:10.1098/rsta.1948. 0007

[25] C. Tannous, J. Gieraltowski, A stoner-wohlfarth model redux: Dynamic and statistical properties, Physica B: Condensed Matter 403 (19-20) (2008) 3578-3584. doi:10.1016/j.physb. 2008.05 .033

[26] R. Street, J. C. Woolley, A study of magnetic viscosity Proceedings of the Physical Society. Section A 62 (9) (1949) 562. URL http://stacks.iop.org/0370-1298/62/i=9/a= 303

[27] C. Tannous, J. Gieraltowski, The stoner-wohlfarth model of ferromagnetism: Static properties (2006). arXiv:http:// arxiv.org/abs/physics/0607117v3

[28] M. F. de Campos, S. A. Romero, F. J. G. Landgraf, F. P. Missell, Estimate of the anisotropy field in isotropic SmCo 2:17 magnets with the stoner-wohlfarth CLC model, Journal of Physics: Conference Series 303 (2011) 012049. doi : 10.1088/ 1742-6596/303/1/012049

[29] X. Xu, Stoner wohlfarth model for magneto anisotropy, Tech. rep. (2016).

[30] G. Friedman, I. Mayergoyz, Stoner-wohlfarth hysteresis model with stochastic input as a model of viscosity in magnetic materials, IEEE Transactions on Magnetics 28 (5) (1992) 2262-2264. doi:10.1109/20.179463

[31] M. F. de Campos, F. A. da Silva, J. A. de Castro, Stonerwohlfarth model for nanocrystalline anisotropic $\mathrm{SM}_{2} \mathrm{CO}_{17}$, Materials Science Forum 775-776 (2014) 431-436. doi:10. 4028/www.scientific.net/msf.775-776.431

[32] C. Tannous, J. Gieraltowski, The stoner-wohlfarth model of ferromagnetism European Journal of Physics 29 (3) (2008) 475 . 
URL http://stacks.iop.org/0143-0807/29/i=3/a= 008

[33] R. Skomski, J. Coey, Permanent Magnetism (Studies in Condensed Matter Physics), 1st Edition, Institute of Physics Publishing, Bristol and Philadelphia, 1999.

[34] L. Daniel, O. Hubert, N. Buiron, R. Billardon, Reversible magneto-elastic behavior: A multiscale approach, Journal of the Mechanics and Physics of Solids 56 (3) (2008) 1018-1042. doi:10.1016/j.jmps.2007.06.003

[35] L. Daniel, M. Rekik, O. Hubert, A multiscale model for magneto-elastic behaviour including hysteresis effects Archive of Applied Mechanics 84 (9) (2014) 1307-1323. doi:10. 1007/s00419-014-0863-9

URL https://doi.org/10.1007/s00419-014-0863-9

[36] N. Buiron, L. Hirsinger, R. Billardon, A multiscale model of magnetostriction strain and stress effect, Journal of Magnetism and Magnetic Materials 226-230 (2001) 1002-1004. doi:10. 1016/s0304-8853(00)01172-0

[37] L. Daniel, O. Hubert, R. Billardon, Homogenisation of magneto-elastic behaviour: from the grain to the macro scale Computational and Applied Mathematics 23 (2-3) (2004) 285-308. doi:10.1590/s1807-03022004000200010 URL http://www.scielo.br/scielo.php?script=sci_ arttext\&pid=S1807-03022004000200010\&lng=en\&nrm= iso>. ISSN2238-3603

[38] A. van den Berg, L. Dupre, B. V. de Wiele, G. Crevecoeur, A mesoscopic hysteresis model based on the unconstrained minimization of the gibbs free energy, IEEE Transactions on Magnetics 46 (2) (2010) 220-223. doi:10.1109/tmag.2009. 2031978

[39] D. Vanoost, S. Steentjes, J. Peuteman, G. Gielen, H. D. Gersem, D. Pissoort, K. Hameyer, Magnetic hysteresis at the domain scale of a multi-scale material model for magneto-elastic behaviour, Journal of Magnetism and Magnetic Materials 414 (2016) 168-179. doi:10.1016/j.jmmm.2016.04.028

[40] S. Ito, T. Mifune, T. Matsuo, C. Kaido, Energy-based magnetization and magnetostriction modeling of grain-oriented silicon steel under vectorial excitations, IEEE Transactions on Magnetics 52 (5) (2016) 1-4. doi:10.1109/tmag. 2016.2519602

[41] F. Martin, U. Aydin, A. Ruzibaev, Y. Ge, L. Daniel, L. Bernard, P. Rasilo, A. Benabou, A. Belahcen, Analysis of the magnetomechanical anisotropy of steel sheets in electrical applications, IEEE Transactions on Magnetics 56 (2) (2020) 1-4. doi:10. 1109/tmag. 2019.2945181

[42] W. D. Armstrong, A directional magnetization potential based model of magnetoelastic hysteresis, Journal of Applied Physics 91 (4) (2002) 2202-2210. doi:10.1063/1.1431433

[43] L. Daniel, N. Galopin, A constitutive law for magnetostrictive materials and its application to terfenol-d single and polycrystals, The European Physical Journal Applied Physics 42 (2) (2008) 153-159. doi:10.1051/epjap:2008031

[44] R. Street, J. C. Woolley, P. B. Smith, Magnetic viscosity under discontinuously and continuously variable field conditions Proceedings of the Physical Society. Section B 65 (9) (1952) 679.

URL http://stacks.iop.org/0370-1301/65/i=9/a= 305

[45] P. Gaunt, Domain wall pinning as a source of magnetic hardening in alloys, Journal of Applied Physics 43 (2) (1972) 637-638. doi:10.1063/1.1661169

[46] P. Gaunt, G. J. Roy, Magnetic viscosity in ferromagnets, Philosophical Magazine 34 (5) (1976) 781-788. doi:10.1080/ 14786437608222050

[47] P. Gaunt, Magnetic viscosity and thermal activation energy, Journal of Applied Physics 59 (12) (1986) 4129-4132. doi :
10.1063/1.336671

[48] L. Lanci, D. V. Kent, Introduction of thermal activation in forward modeling of hysteresis loops for single-domain magnetic particles and implications for the interpretation of the day diagram, Journal of Geophysical Research: Solid Earth 108 (B3) (mar 2003). doi:10.1029/2001jb000944

[49] R. Grössinger, J. Téllez-Blanco, R. S. Turtelli, R. Hauser, K. Reiterer, H. Sassik, G. Chouteau, Determination of the magnetic viscosity in SmCo5-xCux alloys by pulsed-field method, Physica B: Condensed Matter 294-295 (2001) 194-198. doi:10. 1016/s0921-4526(00) 00642-6

[50] J. C. Martinez, F. P. Missell, Magnetic viscosity in NdFeB magnets, Le Journal de Physique Colloques 49 (C8) (1988) C8-649C8-650. doi:10.1051/jphyscol:19888294

[51] J. Liu, H. Luo, S. Pan, Magnetic viscosity studies of Nd16Fe77B7 permanent magnets, Journal of Applied Physics 69 (8) (1991) 5557-5558. doi:10.1063/1.347948

[52] T. Woodcock, Y. Zhang, G. Hrkac, G. Ciuta, N. Dempsey, T. Schrefl, O. Gutfleisch, D. Givord, Understanding the microstructure and coercivity of high performance NdFeB-based magnets, Scripta Materialia 67 (6) (2012) 536-541. doi: 10.1016/j.scriptamat.2012.05.038

[53] S. Bance, J. Fischbacher, A. Kovacs, H. Oezelt, F. Reichel, T. Schrefl, Thermal activation in permanent magnets, JOM 67 (6) (2015) 1350-1356. doi:10.1007/ s11837-015-1415-7

[54] S. Bance, J. Fischbacher, T. Schrefl, Thermally activated coercivity in core-shell permanent magnets, Journal of Applied Physics 117 (17) (2015) 17A733. doi:10.1063/1.4916542

[55] H. Kurose, M. Ohtake, D. Miyagi, N. Takahashi, 3-d FEM analysis of thermal degradation in writing and reading characteristics of a perpendicular magnetic head, Journal of Magnetism and Magnetic Materials 320 (22) (2008) 2917-2920. doi:10.1016/j.jmmm.2008.08.001

[56] L. Bernard, L. Daniel, Effect of stress on magnetic hysteresis losses in a switched reluctance motor: Application to stator and rotor shrink fitting, IEEE Transactions on Magnetics 51 (9) (2015) 1-13. doi:10.1109/tmag.2015.2435701

[57] N. Buiron, L. Hirsinger, R. Billardon, A multiscale model for magneto-elastic couplings, Le Journal de Physique IV 09 (PR9) (1999) Pr9-187-Pr9-196. doi:10.1051/jp4:1999919

[58] N. Buiron, Modélisation multiéchelle du comportement magnéto-élastique couplé des matériaux ferromagnétiques doux, PhD Thesis, École Normale Supérieure de Cachan (2000).

[59] F. Fiorillo, Measurement and Characterization of Magnetic Materials, Elsevier, 2004. doi:10.1016/ B978-0-12-257251-7.X5000-X

[60] L. Bernard, B. J. Mailhé, N. Sadowski, N. J. Batistela, L. Daniel, Multiscale approaches for magneto-elasticity in device simulation, Journal of Magnetism and Magnetic Materials 487 (2019) 165241. doi:10.1016/j.jmmm.2019.04.093

[61] J. C. Martinez, F. P. Missell, Magnetic viscosity and texture in NdFeB magnets, Journal of Applied Physics 64 (10) (1988) 5726-5728. doi:10.1063/1.342239

[62] O. Engler, V. Randle, Introduction to Texture Analysis: Macrotexture, Microtexture, and Orientation Mapping, 2nd Edition, CRC Press, 2009.

[63] H. Hauser, Energetic model of ferromagnetic hysteresis, Journal of Applied Physics 75 (5) (1994) 2584-2597. doi:10.1063/ 1.356233

[64] D. R. Cornejo, A. Azevedo, S. M. Rezende, Hysteresis modeling of anisotropic and isotropic nanocrystalline hard magnetic films, Journal of Applied Physics 93 (10) (2003) 6623-6625. doi: 10.1063/1.1557355

[65] A. Bergqvist, D. Lin, P. Zhou, Temperature-dependent vector 
hysteresis model for permanent magnets, IEEE Transactions on Magnetics 50 (2) (2014) 345-348. doi : 10.1109/tmag. 2013. 2282822

[66] R. Skomski, J. Coey, Nucleation field and energy product of aligned two-phase magnets-progress towards the $1 \mathrm{mj} / \mathrm{m} 3 \mathrm{mag}$ net, IEEE Transactions on Magnetics 29 (6) (1993) 2860-2862. doi:10.1109/20.281077

[67] R. Skomski, J. Zhou, R. D. Kirby, D. J. Sellmyer, Magnetic aging, Mater. Res. Soc. Symp. Proc. 887 (2006).

[68] R. Skomski, Simple Models of Magnetism, Oxford University Press, 2007.

[69] E. P. Wohlfarth, The coefficient of magnetic viscosity Journal of Physics F: Metal Physics 14 (8) (1984) L155.

URL http://stacks.iop.org/0305-4608/14/i=8/a= 005

[70] British Standard, Magnetic materials - Permanent magnet (magnetically hard) materials - Methods of measurement of magnetic properties - BS EN 10332:2003 (2003).

[71] M. Domenjoud, E. Berthelot, N. Galopin, R. Corcolle, Y. Bernard, L. Daniel, Characterization of giant magnetostrictive materials under static stress: influence of loading boundary conditions, Smart Materials and Structures 28 (9) (2019) 095012. doi:10.1088/1361-665x/ab313b 